\title{
Does psychological intervention help chronic skin conditions?
}

\author{
Harbans S Capoore, Christopher M E Rowland Payne, David Goldin
}

\begin{abstract}
Summary
The objective of the study was to assess the impact of psychological/psychiatric assessment in patients with chronic or intractable dermatological conditions. A diagnostic interview was undertaken in order to pin-point any temporal connection between an adverse life-event and the first appearance of the skin disorder. Following this, the dermatologist's assessment of subsequent changes in the skin disorder were noted. The three main dermatological diagnoses were atopic eczema (10), prurigo (10), and psoriasis (nine). Follow-up was for up to 5 years.
\end{abstract}

A favourable response was noted in $\mathbf{4 0}$ out of the 64 patients who participated in the study; this was usually evident within a few weeks and maintained thereafter. Remission was achieved in 12, while 28 showed some improvement. We conclude that this liaison between dermatology and psychiatry proved a valuable adjunct to normal dermatological treatment and was followed by improvement in the majority of patients.

Keywords: psychosomatic medicine; skin disorders

Patients with chronic or intractable dermatoses may suffer from psychological/psychiatric comorbidity. There is no published work relating to a precise psychological event or events underlying the onset of a dermatosis and this study was set up to examine this possibility.

Illness may be studied in two ways, firstly by considering the recognised and established patterns in organic and psychological disorders, and secondly by taking into account temporal relationships in the onset and development of the disorder; something to understand rather than categorize.

In this latter context, an adverse life event may be important in understanding the mode of onset of the illness. Such an event may be one that the individual has construed as being threatening, damaging, or even dangerous, and for which there appears to be no solution.

This experience of an adverse life event may result in distress and or conflict leading to mental or physical change or a combination of the two. ${ }^{2}$ In the latter instance it has become customary to speak of a psychosomatic disorder. ${ }^{3}$ The degree of importance attaching to either component varies and each needs assessing carefully. Thus, we have to consider:
- events associated with the onset of the skin disorder

- traumatic events occurring just before or about the time of onset of skin disorder

- evidence of psychiatric disorder both before and subsequent to the appearance of the skin disorder.

\section{Methods}

The aims and objective were to determine whether life events had been causally related to the onset of the dermatological condition and to determine whether psychiatric intervention had led to an improvement in the dermatosis. In total, 64 patients with chronic or intractable skin conditions were invited to participate. Patients were of both sexes (28 men, 36 women, age range 3-81 years) and were attending the dermatology out-patient clinic. The criteria for selection used by the dermatologists were based on clinical need, ie, the patient suffered from a chronic or intractable condition, or one resistant to conventional treatment. Paradoxically, certain diseases such as dermatitis artefacta and pruritus vulvae, customarily considered to be psychosomatic, comprised a minority of cases. The patients were recruited over 3 years and followed-up for up to 5 years.

Each subject was asked by their dermatologist if they would agree to having a talk with one of the team (HSC), with expertise and interest in the emotional problems connected with skin disorders. After the talk they would be seen again by the dermatologist. Wherever possible, a family member was present. The patient was informed that the aim was to take a medical history to see if there were any other factors affecting their condition, that they need not talk about anything they did not wish to, and that all information would be treated as confidential. In the case of a child, the mother was questioned and the child asked to join in the discussion whenever he or she wished.

A brief life-history was obtained in all cases. The emphasis was on the previous health of the patient or members of the immediate family, death of anyone close to the patient, difficulties experienced during different periods of their life, their relationships with others in the family, at school or at work, problems with friends, their hopes and ambitions. A mother's undue concern for her child, learning difficulties (due to visual or auditory disability or dyslexia) and unsatisfactory scholastic achievement, stability of marriage, change in job 


\begin{tabular}{|l|}
\hline Significant life events \\
\hline In adults: \\
- retirement and divorce or separation from spouse \\
- isolation, particularly in old age \\
- incapacitating illness in patient or close relative \\
- moving to new surroundings \\
- a bereavement seriously affecting the patient \\
In children and young patients: \\
- absence of mother \\
- difficult home conditions \\
- serious school difficulties, eg, hearing disability \\
- chronic illness \\
\hline
\end{tabular}

Box 1

conditions, adjustment to retirement, isolation and loneliness may be of importance. If a possible connection between an event and the onset of the skin disorder was identified, its importance was discussed briefly with the patient. Each patient also underwent a psychiatric assessment to look for the presence / a history of psychiatric illness.

The salient features of the history were reported to the dermatologist, who saw the patient again and concluded the consultation, in order to reassure the patient that their problem was dermatological.

All assessments as to outcome were carried out by the responsible dermatologist in simple terms of remitted, improved, and not improved or on-going. They were based on his previous experience with the patient, estimation of the degree of reduction of symptoms and disability, the ability to return to normal activities and the quality of life of the patient. The psychiatrist was not involved in the final assessment.

\section{Results}

A significant life event was identified in 44 patients; this was usually associated with a good outcome. The most common life events in adults are listed in box 1.

The outcome was favourable in 40 out of the total of 64 patients (table 1). A favourable outcome was classified as remitted (full and satisfactory response leading to early discharge), or improved (condition improved but discharge without supervision not possible). The outcome in relation to the dermatological diagnosis was of interest (table 1). Atopic eczema, prurigo and psoriasis ${ }^{4}$ are probably some of the commonest skin disorders and these patients tended to respond favourably to our psycho-

Table 1 Patient details

\begin{tabular}{lccccc}
\hline $\begin{array}{l}\text { Dermatological } \\
\text { diagnosis }\end{array}$ & $\begin{array}{l}\text { No of } \\
\text { patients }\end{array}$ & $\begin{array}{l}\text { Sex } \\
\text { (F:M) }\end{array}$ & $\begin{array}{l}\text { Age range } \\
\text { (years) }\end{array}$ & Remitted & Improved \\
\hline Atopic eczema & 10 & $4: 6$ & $3-71$ & 4 & 5 \\
Prurigo & 10 & $5: 5$ & $7-81$ & - & 8 \\
Psoriasis & 9 & $5: 4$ & $10-69$ & 1 & 4 \\
Others & 35 & $22: 13$ & $11-78$ & 7 & 11 \\
\hline
\end{tabular}

Table 2 Psychological diagnosis ${ }^{5}$

\begin{tabular}{lr}
\hline Diagnosis & $n$ \\
\hline Psychosis: & 6 \\
Major depressive disorder & 1 \\
Delusional disorder & 18 \\
Minor depressive or anxiety disorder & 2 \\
Bereavement & 8 \\
Psychosocial problems & 9 \\
Clinical disorder (ie, psychological concern over a & \\
medical illness) & 20 \\
No diagnosis &
\end{tabular}

logical intervention (22 patients). The remaining 35 patients had diagnoses belonging to one of 22 other categories, mainly single cases such as acne, dermatitis artefacta, alopecia areata, discoid eczema, and rosacea. About half of these patients responded favourably (18/35 patients).

Psychological diagnoses ${ }^{5}$ (table 2) showed the usual range of disorders seen in a group of patients, with the largest number being in the minor depressive and anxiety category. However, no psychological diagnosis was evident in 20 patients. Psychoses were found in seven patients. Psychological diagnoses were scattered among the various dermatological diagnoses without any particular pattern. No relationship was identified between the psychological diagnosis and dermatological diagnosis.

The speed and durability of improvement after the intervention was notable in a number of patients. This outcome was all the more encouraging by its unexpectedness and confounded the scepticism of the dermatologists involved.

\section{Discussion}

There is nothing new in the idea that emotions can profoundly influence physiological activity and result in dysfunction. Mention of this possibility was put forward in a dermatology textbook as early as $1957 .^{6}$ There may be a continuing physiological change, which eventually becomes a semi-permanent feature of the patient's life. Failure to remember may be due to the patient's need not to recognise or admit to emotional difficulties.

We concentrated on uncovering the actual life event or events that occurred at or about the time of onset of the skin condition. There was no attempt to explore or explain the feelings of the patient at the time or subsequently. The aim was to demonstrate that the intervention had led to a change in the patient's dermatological condition. In patients showing a good response, a new optimism emerged rapidly and they were both satisfied and confident. Enquiry from a relative or friend confirmed this impression and re-referral rarely occurred.

Two factors seemed to be associated with a poor response to intervention: habit formation, and secondary gain, ${ }^{7}$ when the continuation of the skin disorder serves purposes which are advantageous to the patient. The duration of the skin disorder was not of itself a bad prognostic sign, but if combined with either one or both of the above factors, it could be. 
The possible factors contributing to improvement are listed in box 2 . By increased insight we mean enhanced self-knowledge and a better understanding by the patient of circumstances prevailing at the time of the onset of the skin disorder.

As most of the 64 patients had already proved refractory to the usual dermatological therapies this outcome can be regarded as a substantial success.

The conclusion that emerges from this study is that traumatic experience had continued to affect the emotional state of nearly two-thirds of this group of dermatological patients and this in turn played a major role in the progress of the disorder and its resistance to treatment.

Such a psychological/psychiatric intervention could usefully be included in the normal dermatology assessment, as it should both improve patient care and be cost effective. To our knowledge this is the largest series yet from

1 Brown GW, Harris T, eds. Life events and illness. New York Guildford Press, 1989.

2 Chen CC, David AS, Nunnerly H, et al. Adverse life events and breast cancer: case control study. BMF 1995;311:1527-

3 Mcrimmon RJ. Psychosomatic syndromes: Report of a symposium held in the College on 5 April 1995. Proc $R$ Coll posium held in the College on 5 April
Physicians Edinburgh 1996;26:36-40.

4 Payne RA. Rowland Payne CME, Marks R. Stress does not Payne RA. Rowland Payne CME, Marks R. Stress does not
worsen psoriasis? A controlled study of 32 patients. Clin Exp Dermatol 1985;10:239-45.

\section{Factors contributing to improvements}

- opportunity to talk and ask questions

- attention received

- evidence of emotional reaction during interview

- the power of suggestion

- placebo effect

- increased insight

Box 2

a dermatology-psychiatry liaison clinic. The procedure described above has a simplicity and commonality which may recommend it for further consideration and research (and not only in the dermatological setting).

We are grateful to Dr A F Muller for his helpful comments on the paper.

5 American Psychiatric Association (APA). Mental disorders, 4th edn (DSM-IV). Washington DC: APA, 1994.

6 Ingram JT, Brain RT. Sequeira's diseases of the skin, 6th edn London: Churchill, 1957; pp 1-2.

7 Craig TKJ, Drake H, Mills K, Boardman AP. The South London somatization Study II. Influence of stressful life events and secondary gain. Br $\mathcal{F}$ Psychiatry 1994;165:24858. 